\title{
Developing a Vis/NIR spectroscopic system for fast and non-destructive pesticide residue monitoring in agricultural product
}

\author{
Bahareh Jamshidi $^{*}$, Ezeddin Mohajerani ${ }^{\mathrm{b}}$, Jamshid Jamshidi $^{\mathrm{c}}$ \\ ${ }^{a}$ Agricultural Engineering Research Institute, Agricultural Research Education and Extension \\ Organization (AREEO), Karaj, Iran; \\ bLaser and Plasma Research Institute, Shahid Beheshti University, Tehran, Iran; \\ 'Zarif Mosavar Industrial Manufacturing Company, Isfahan, Iran;
}

*Corresponding author: Bahareh Jamshidi, E-mail: b.jamshidi@areo.ir, Tel: +98 2632705320, Fax: +98 2632706277

\begin{abstract}
In this research, an optical system based on fibre optic Vis/NIR spectroscopy combined with chemometrics methods and software as a graphical user interface (GUI) was developed and presented for fast and non-destructive detection and determination of pesticide residues in agricultural products (a case study on diazinon in intact cucumbers). Vis/NIR spectra of cucumber samples without and with different concentrations of diazinon residue were analyzed at the range of 450-1000 $\mathrm{nm}$. Partial least squares (PLS) regression models were developed based on chemical reference measurements and the spectral information of the samples after performing different pre-processing methods. Moreover, partial least squares-discriminant analysis (PLS-DA) models were developed based on different spectral pre-processing techniques to classify cucumbers with contents of diazinon below and above the maximum residue limits (MRL) as safe and unsafe samples, respectively. Finally, user-friendly software as a GUI was created based on the best PLS and PLS-DA models developed for prediction of diazinon contents in the samples and for classification of intact cucumbers by the absence/presence of diazinon residues, respectively. Evaluation of the system and software designed based on the best developed PLS and PLS-DA models indicated good performance for measuring and detection of
\end{abstract}


diazinon residue in cucumbers. It was concluded that the designed system and software based on Vis/NIR spectroscopy combined with chemometrics methods can be utilized for fast and nondestructive safety control of intact cucumbers by the absence/presence of diazinon residues. It can also be generalized for detection of other pesticide residues in agricultural products if developing their appropriate models is feasible.

Keywords: Chemometrics; GUI; Non-destructive; Pesticide Residues; Safety; Vis/NIR Spectroscopy

\section{Introduction}

Typically, the detection of pesticides in complex matrices, such as agricultural products, involves a sample treatment using different methods such as solid-phase extraction, supercritical fluid extraction, microwave-assisted extraction, and accelerated solvent extraction [1, 2]. Conventional techniques such as gas chromatography [3], high-performance liquid chromatography [4], thin-layer chromatography [5], supercritical fluid chromatography [6], gas and liquid chromatography combined with mass spectrometry [5, 7], capillary electrophoresis [8, 9], enzyme inhibition method [5, 10], immunoassay method [5, 11], and bio-sensor method [5, 12], are also used to measure the concentration of pesticide residue in agricultural products. However, these techniques are destructive, difficult, highly time consuming, very expensive, environmentally unfriendly, and enable safety control of a few samples per batch as well as require sample preparation, well-trained personnel and advance laboratory. Therefore, there is a need for development of a non-destructive, simple, fast, low-cost, environmentally friendly with little sample preparation, and reliable detection technique of pesticide residue in agricultural products for controlling each individual sample [2, 13].

One of the most promising non-destructive techniques is Near-infrared (NIR) spectroscopy which has been successfully applied for qualitative and quantitative analyses especially to quality control of intact fruits and vegetables [14-22]. This technique has non-contaminant and lossless nature, the low operating cost compared with the conventional techniques, and the fast response times. It also requires little or no sample preparation and can be used in processing lines [23]. There are also some researches about detection or determination of pesticide residues in such 
products [1, 2, 24-27]. All of these researches show the feasibility of using NIR spectroscopy for detection of pesticide residues.

This research aims to develop a visible/near-infrared (Vis/NIR) Spectroscopic system for fast and non-destructive safety control of agricultural products based on absence/presence of pesticide residues with a case study on diazinon detection and determination in cucumber. To this end, the feasibility of utilizing Vis/NIR spectroscopy combined with partial least squares (PLS) regression and different spectral pre-processing methods were investigated for measuring the residue content in the samples non-destructively. Moreover, the results reported by Jamshidi et al. [2], who have assessed the feasibility of using this technique combined with partial least squares-discriminant analysis (PLS-DA) for non-destructive classification of intact cucumbers by the absence/presence of diazinon residues based on containing the levels below/above the maximum residue limits (MRL), $0.1 \mathrm{mg} \mathrm{kg}^{-1}$, established by FAO/WHO Codex Alimentarius [28], were used. Then, user-friendly software as a graphical user interface (GUI) was designed based on the best PLS and PLS-DA models developed for prediction of diazinon contents in the samples and for classification of intact cucumbers by the absence/presence of diazinon residues, respectively.

\section{Materials and Methods}

\subsection{Cucumbers preparation}

A total of 120 cucumbers were harvested from the greenhouses near Karaj city in Alborz province of Iran. The $60 \%$ diazinon pesticide $\left(\mathrm{C}_{12} \mathrm{H}_{21} \mathrm{~N}_{2} \mathrm{O}_{3} \mathrm{PS}\right)$ was used to obtain different pesticide residue contents in some samples as a pesticide-contaminated set. It was diluted to 1/500 and sprayed on some of the cucumbers. Some other samples were placed in the prepared solution for $1 \mathrm{~h}$. A pesticide-free set of cucumbers was also used. All samples were stored at $5^{\circ} \mathrm{C}$ until Vis/NIR spectroscopy.

\subsection{Experiments}

Before each experiment, cucumbers were left until their temperature had risen to the laboratory temperature. Vis/NIR measurements of the samples at the range of 450-1000nm were conducted with a USB2000 fibre optic spectrometer (Oceanoptics Inc., USA), in interactance mode. Before spectra acquisition of the samples, white reference and dark spectra were collected to obtain the relative interactance. For each cucumber, Vis/NIR spectra at thirty scans from different positions 
on the sample were acquired by OOIBase32 software (Oceanoptics). The mean spectrum was calculated for each sample and stored for later data analysis.

After each spectroscopy experiment, the cucumbers were sent to the Chemical Analysis Center at Iranian Institute of R\&D in Chemical Industries for measurement of diazinon content in each sample with a reference method, GC analysis. To this end, sample preparation was done based on British Standard BS EN 15662:2008 [29]. Then, an Agilent 7890A gas chromatograph (Agilent Technologies Inc., Santa Clara, CA, USA) was used to measure the concentration of pesticide residue in each sample [2].

\subsection{PLS analysis}

The overall Vis/NIR spectra of the samples were converted to absorbance values $(\log (1 / R))$. Principal component analysis (PCA) was used to determine the outlier samples. After removing the outliers (14 samples), all the 106 remained samples were utilized for quantitative analysis in order to measure the diazinon content.

PLS regression models were developed based on GC measurements and the spectral information of the cucumbers. Before modeling, multiplicative scatter correction (MSC) and standard normal variate (SNV) were used to correct both multiplicative and additive effects of the Vis/NIR spectra. First and second derivatives of the spectra $\left(D_{1}, D_{2}\right)$ were also performed to increase the spectral resolution. The algorithm used most often for derivation is Savitzky-Golay. The data within a moving window are fitted by a polynomial of a given degree. Therefore, it is important to select the proper width of the moving window. In general, it should not exceed one point five times as the half width of the absorbance peak in the spectra [30]. To this end, Savitzky-Golay algorithm with five smoothing points and polynomial order of two was used for derivation.

Then, PLS models were developed with full cross-validation and investigating the maximum of 10 latent variables (LVs). Comparison of the calibration models was done based on having the lowest standard error of cross-validation (SECV) and the highest correlation coefficient of cross validation $\left(\mathrm{r}_{\mathrm{cv}}\right)$. All analyses were conducted using the Unscrambler software X10.3 (CAMO Software AS, Norway).

\subsection{Software design}

To complete the spectroscopic system for safety assessment of the cucumbers, the best PLS model developed for prediction of diazinon contents in the samples was used. Moreover, the best PLS-DA model developed by Jamshidi et al. [2] was also used to discriminate the safe samples 
(all samples without or with diazinon concentrations $\leq 0.1 \mathrm{mg} \mathrm{kg}^{-1}$ ) from the unsafe cucumbers (all samples with diazinon concentrations $>0.1 \mathrm{mg} \mathrm{kg}^{-1}$ ), respectively. Therefore, user-friendly software as a GUI was created based on the best PLS and PLS-DA models to predict diazinon contents in the samples and to classify the cucumbers by the absence/presence of diazinon residues, respectively.

This computer software was created in Microsoft visual studio 2013 (Microsoft, USA), a rich integrated development environment, using Microsoft visual basic programming language. To build a modern line-of-business desktop application on windows, windows presentation foundation (WPF) in visual studio (.NET framework 4) was also used [31]. Figure 1 shows the software flowchart.

Performance of the system and software designed based on the best developed PLS and PLS-DA models was evaluated for the samples of validation set in qualitative analysis (26 samples) [2]. Figure 2 shows the presented system for non-destructive safety assessment of the cucumbers. The paired t-test was performed in Minitab17 statistical software (Minitab Inc., USA) to investigate the difference of means between diazinon values predicted with the designed system and the measured values using GC analysis.

\section{Results and Discussion}

\subsection{Statistics}

The cucumbers were quite varied in terms of morphological properties including the weight, maximum equatorial diameter, and the length of the samples [2]. The statistics of diazinon contents in the samples are shown in Table 1. As can be seen, there was a large variability in the diazinon concentrations of the cucumbers ranging 0-32 $\mathrm{mg} \mathrm{kg}^{-1}$ including both safe and unsafe samples. The mean and standard deviation (SD) of diazinon contents in total samples were 4.85 and $7.44 \mathrm{mg} \mathrm{kg}^{-1}$, respectively. There were 21 cucumbers without or with diazinon concentrations $\leq 0.1 \mathrm{mg} \mathrm{kg}^{-1}$ as safe samples. The remained 85 samples were unsafe which had diazinon concentrations $>0.1 \mathrm{mg} \mathrm{kg}^{-1}$. The SD of diazinon contents in safe and unsafe samples were 0.03 and $8.22 \mathrm{mg} \mathrm{kg}^{-1}$, respectively.

\subsection{Vis/NIR spectra}

The mean spectra $(\log (1 / \mathrm{R}))$ of intact cucumbers with absence of pesticide (AP) and presence of pesticide (PP) are presented in Figure 3. The difference between the spectra was observed in 
both visible and NIR regions. In the visible region, there was a peak around 680nm due to the chlorophyll absorbance in both samples of AP and PP. However, presence of pesticide in the sample caused decrease of this absorbance. Increase of the absorbance in NIR region after 900 $\mathrm{nm}$ for both samples of AP and PP could be due to the second overtone of O-H or the third overtone of $\mathrm{C}-\mathrm{H}$. It was noted that the increase of the absorbance in this region for the pesticidecontaminated samples is more than that in the pesticide-free cucumbers. According to that reported by other researchers [2, 26, 27], this increase is more related to $\mathrm{C}-\mathrm{H}$ absorbance.

\subsection{Preprocessing and multivariate analysis}

Table 2 shows the results of PLS models developed with different pre-processing methods for prediction of diazinon residue in the cucumbers. All the developed calibration models had acceptable ability for prediction of diazinon residue in the samples with $r_{c v}$ above 0.80 . However, the best prediction results were achieved using PLS model developed based on MSC+D 1 method $\left(r_{c v}=0.91, S E C V=3.22\right)$. The predicted versus reference values for this calibration model is presented in Figure 4.

The obtained results in this research were better than those achieved by Saranwong and Kawano [27] who used a dry-extract system for infrared analysis (DESIR) technique to detect dichlofluanid fungicide levels on the surface of tomato using a PLS model developed based on $\mathrm{D}_{2}$ pre-processing technique ( $\left.\mathrm{SEP}=7.89\right)$. These results were also better than those reported by Xue et al. [1] who assessed the feasibility of Vis/NIR spectroscopy in the range of 350-1800 nm to determine dichlorvos residue on the surface of navel orange using a PLS model developed based on particle swarm optimization (PSO) algorithm ( $\mathrm{r}=0.87$ ).

Therefore, the regression coefficients of the best developed model using PLS and MSC $+\mathrm{D}_{1}$ was used to create the user-friendly software as a GUI for prediction of diazinon contents in the samples (Fig. 5). Besides, the results of the best PLS-DA model developed by Jamshidi et al. [2] based on $\mathrm{D}_{1}$ pre-processing technique $(\mathrm{SECV}=0.366$, total percentages of correctly classified samples $=97.5 \%$ ) was utilized to classify intact cucumbers by the absence/presence of diazinon residues in the GUI.

\subsection{Software performance}

According to Figure 1, the first stage to create GUI was reading data, the raw spectra of intact sample obtained using the Vis/NIR spectroscopic system in measurement mode of interactance (up to 20 spectra from the different positions on the sample). Then, some calculations such as 
converting the reflectance to the absorbance values, baseline correction, creating the appropriate spectral region from 450 to $1000 \mathrm{~nm}$, and averaging the spectra were done to prepare the spectra for displaying and the next analysis. After that, the mean spectrum was pre-processed using $\mathrm{D}_{1}$ because this technique caused the best results of both PLS and PLS-DA models developed for diazinon prediction and discrimination of the safe and unsafe samples, respectively. The class of the cucumber was identified using the results of the PLS-DA analysis [2]. If the sample was belonged to the safe group, GUI displayed 'safe' for the class of the sample and ' $\leq 0.1$ ' for the predicted value of diazinon. For a cucumber that was belonged to unsafe group, diazinon content was predicted using the PLS model. GUI displayed 'unsafe' for the class and the predicted value of diazinon, if it was above the MRL $\left(0.1 \mathrm{mg} \mathrm{kg}^{-1}\right)$. Sometimes, PLS model may incorrectly predict the residue content of the unsafe sample below the MRL. Moreover, a safe sample may be misclassified into unsafe group using the PLS-DA model when the PLS model correctly predicts the residue content below the MRL. In these cases, GUI displayed the result of 'unpredictable, repeat' to inform user about the error between the results of two PLS and PLSDA models and to repeat the test. Figure $6(a-b)$ shows the performance of the designed GUI for safety assessment of two typical cucumbers without and with concentrations of diazinon residue. Evaluation of the system for the 26 samples, external validation set in PLS-DA analysis containing 5 safe and 21 unsafe samples, indicated that the system and the software had very good ability to classify the unknown cucumbers. The total percentage of correctly classified samples was $92.31 \%$. It was noted that all the samples of unsafe group were $100 \%$ correctly classified and the percentage of correctly classified cucumbers of safe group was $60 \%$. While two samples of safe group were misclassified into the unsafe group, diazinon content in one of them was predicted below MRL using the PLS predictor model and the GUI displayed the result of 'unpredictable, repeat'. According to the prediction results for diazinon content of the samples of unsafe group using the system, GUI could display the predicted value for 17 samples and the result of 'unpredictable, repeat' for the remained cucumbers (4 samples). Paired t-test results (Table 4) demonstrated that the mean values of diazinon residue predicted with the designed system and the measured values using GC analysis were not significantly different $(\mathrm{p}>0.05)$.

\section{Conclusions}


A Vis/NIR spectroscopic system based on multivariate qualitative and quantitative models and software as a GUI was developed and presented for fast discrimination of intact cucumbers by presence/absence of diazinon residue and for prediction of the residue content in the samples non-destructively. The obtained results confirmed the potential of the designed system for classification of intact cucumbers with contents of diazinon below and above the MRL as safe and unsafe samples, respectively. The system had also good ability to predict the residue content in the unsafe samples with no significant difference $(p>0.05)$ between the mean values of diazinon residue predicted and measured using the system and reference method, respectively. Therefore, the developed system could be used for fast, low-cost and non-destructive preliminary screening of the cucumbers in terms of contaminating by pesticide residues as a portable system. A noticeable result is the capability of the created GUI to be easily learned and used by user, to respond rapidly, to change the spectral region based on type of the spectrometer which is used, to be compatible and flexible to the different situations and needs, and to be generalized for detection of other pesticide residues in other fruits and vegetables if developing their appropriate models is feasible.

\section{Acknowledgments}

This research was funded by Iran National Science Foundation (INSF), project No. 91051000, and supported by Agricultural Engineering Research Institute as well as Laser \& Plasma Institute of Shahid Beheshti University. The authors are grateful to the Chemical Analysis Center at Iranian Institute of R\&D in Chemical Industries for the cooperation on reference measurements.

\section{References}

[1] L. Xue, J. Cai, J. Li, M. Liu, Application of particle swarm optimization (PSO) algorithm to determine dichlorvos residue on the surface of navel orange with Vis-NIR spectroscopy, Procedia Eng, 29 (2012) 4124-4128.

[2] B. Jamshidi, E. Mohajerani, J. Jamshidi, S. Minaei, A. Sharifi, Non-destructive detection of pesticide residues in cucumber using visible/near-infrared spectroscopy, Food Addit Contam Part A, 32 (2015) 857-863. 
[3] S.K. Cho, A.M. Abd El-Aty, M. Musfiqur Rahman, J.H. Choi, J.H. Shim, Simultaneous multi-determination and transfer of eight pesticide residues from green tea leaves to infusion using gas chromatography, Food Chem, 165 (2014) 532-539.

[4] E. Watanabe, Y. Kobara, K. Baba, H. Eun, Determination of seven neonicotinoid insecticides in cucumber and eggplant by water-based extraction and high-performance liquid chromatography, Anal Lett, 48 (2015) 213-220.

[5] W. Li, M. Sun, M. Li, A survey of determination for organophosphorus pesticide residue in agricultural products, Adv J Food Sci Technol, 5 (2013) 381-386.

[6] Y. Tao, F. Dong, J. Xu, X. Liu, Y. Cheng, N. Liu, Z. Chen, Y. Zheng, Green and sensitive supercritical fluid chromatographic-tandem mass spectrometric method for the separation and determination of flutriafol enantiomers in vegetables, fruits, and soil, J Agric Food Chem, 62 (2014) 11457-11464.

[7] A. Masiá, C. Blasco, Y. Picó, Last trends in pesticide residue determination by liquid chromatography-mass spectrometry, Trends Environ Anal Chem, 2 (2014) 11-24.

[8] A. Juan-García, G. Font, C. Juan, Y. Pico, Pressurised liquid extraction and capillary electrophoresis-mass spectrometry for the analysis of pesticide residues in fruits from Valencian markets, Spain, Food Chem, 120 (2010) 1242-1249.

[9] A. Juan-García, Y. Pico, G. Font, Capillary electrophoresis for analyzing pesticides in fruit and vegetables using solid-phase extraction and stir-bar sorptive extraction, J Chromatogr A, 1073 (2005) 229-236.

[10] C. Qiu, X. Liu, H. Ren, F. Jiang, Study on detection of organophosphorus pesticide residue in vegetables by enzyme inhibition method, Food Mach, 26 (2010) 40-42.

[11] J.V. Mercader, R. López-Moreno, F.A. Esteve-Turrillas, A. Abad-Somovilla, A. AbadFuentes, Immunoassays for trifloxystrobin analysis. Part II. Assay development and application to residue determination in food, Food Chem, 162 (2014) 41-46.

[12] G. Istamboulie, D. Fournier, J.L. Marty, T. Noguer, Phosphotriesterase: A complementary tool for the selective detection of two organophosphate insecticides: chlorpyrifos and chlorfenvinfos, Talanta, 77 (2009) 1627-1631.

[13] E. Teye, X. Huang, N. Afoakwa, Review on the potential use of near infrared spectroscopy (NIRS) for the measurement of chemical residues in food, Am J Food Sci Technol, 1 (2013) 1-8. 
[14] B. Jamshidi, S. Minaei, E. Mohajerani, H. Ghassemian, Reflectance Vis/NIR spectroscopy for nondestructive taste characterization of Valencia oranges, Comput Electron Agric, 85 (2012) 64-69.

[15] B. Jamshidi, S. Minaei, E. Mohajerani, H. Ghassemian, Prediction of soluble solids in oranges using visible/near-infrared spectroscopy: Effect of peel, Int J Food Properties, 17 (2014) 1460-1468.

[16] S.N. Jha, K. Narsaiah, P. Jaiswal, R. Bhardwaj, M. Gupta, R. Kumar, R. Sharma, Nondestructive prediction of maturity of mango using near infrared spectroscopy, J food Eng, 124 (2014) 152-157.

[17] P. Maniwara, K. Nakano, D. Boonyakiat, S. Ohashi, M. Hiroi, T. Tohyama, The use of visible and near infrared spectroscopy for evaluating passion fruit postharvest quality, J Food Eng, 143 (2014) 33-43.

[18] M.T. Sánchez, M.J. De la Haba, M. Benítez-López, J. Fernández-Novales, A. Garrido-Varo, D. Pérez-Marín, Non-destructive characterization and quality control of intact strawberries based on NIR spectral data, J Food Eng, 110 (2012) 102-108.

[19] I. Torres, D. Pérez-Marín, M.J. De la Haba, M.T. Sánchez, Fast and accurate quality assessment of Raf tomatoes using NIRS technology, Postharvest Biol Technol, 107 (2015) 9-15. [20] R. Vitale, M. Bevilacqua, R. Bucci, A.D. Magrì, A.L. Magrì, F. Marini, A rapid and noninvasive method for authenticating the origin of pistachio samples by NIR spectroscopy and chemometrics, Chemometr Intell Lab Syst, 121 (2013) 90- 99.

[21] H. Wang, J. Peng, C. Xie, Y. Bao, Y. He, Fruit quality evaluation using spectroscopy technology: A review, Sensors, 15 (2015) 11889-11927.

[22] L. Xie, Y. Ying, T. Ying, Rapid determination of ethylene content in tomatoes using visible and short-wave near-infrared spectroscopy and wavelength selection, Chemometr Intell Lab Syst, 97 (2009) 141-145.

[23] Y. Peng, Y. Li, J. Chen, Optical technologies for determination of pesticide residue, In: T. Theophile editor, Infrared spectroscopy - materials science, engineering and technology, InTech, (2012) 453-466.

[24] F. Dai, T. Hong, K. Zhang, Y. Hong, Nondestructive detection of pesticide residue on longan surface based on near infrared spectroscopy, International Conference on Intelligent Computation Technology and Automation (ICICTA), 2 (2010) 781-783. 
[25] L. Salguero-Chaparro, A.J. Gaitán-Jurado, V. Ortiz-Somovilla, F. Peña-Rodríguez, Feasibility of using NIR spectroscopy to detect herbicide residues in intact olives, Food Control, 30 (2013) 504-509.

[26] M.T. Sánchez, K. Flores-Rojas, J.E. Guerrero, A. Garrido-Varo, D. Pérez-Marín, Measurement of pesticide residues in peppers by near-infrared reflectance spectroscopy, Pest Manag Sci, 66 (2010) 580-586.

[27] S. Saranwong, S. Kawano, Rapid determination of fungicide contaminated on tomato surfaces using the DESIR-NIR: A system for ppm-order concentration, J Near Infrared Spectrosc, 13 (2005) 169-175.

[28] Food and Agriculture Organization of the United Nations and World Health Organization (FAO/WHO), Codex Pesticides Residues in Food Online Database, 2013. Available from: http://www.codexalimentarius.net/pestres/data/index.html.

[29] British Standard (BS EN 15662), Foods of plant origin -Determination of pesticide residues using GC-MS and/or LC-MS/MS following acetonitrile extraction/partitioning and clean-up by dispersive SPE - QuEChERS-method, 2008.

[30] H. Cen, Y. He, Theory and application of near infrared reflectance spectroscopy in determination of food quality. Trends Food Sci Technol, 18 (2007) 72-83.

[31] R. Eisenberg, C. Bennage, Sams Teach Yourself WPF in 24 Hours. 1st Reprint. Sams Publishing, United States, 2008. 
Figure 1. Software flowchart.

Figure 2. Safety assessment of the samples with the presented Vis/NIR spectroscopic system.

Figure 3. Mean spectra of intact cucumbers with absence of pesticide (AP) and presence of pesticide (PP).

Figure 4. Scatter plot of the correlation between measured and predicted values of diazinon residue in the samples for full cross validation of calibration set.

Figure 5. The obtained regression coefficients using the best PLS model developed for diazinon content prediction.

Figure 6. The performance result of the created GUI for assessment of the typical cucumbers without (a) and with (b) concentrations of diazinon residue. 


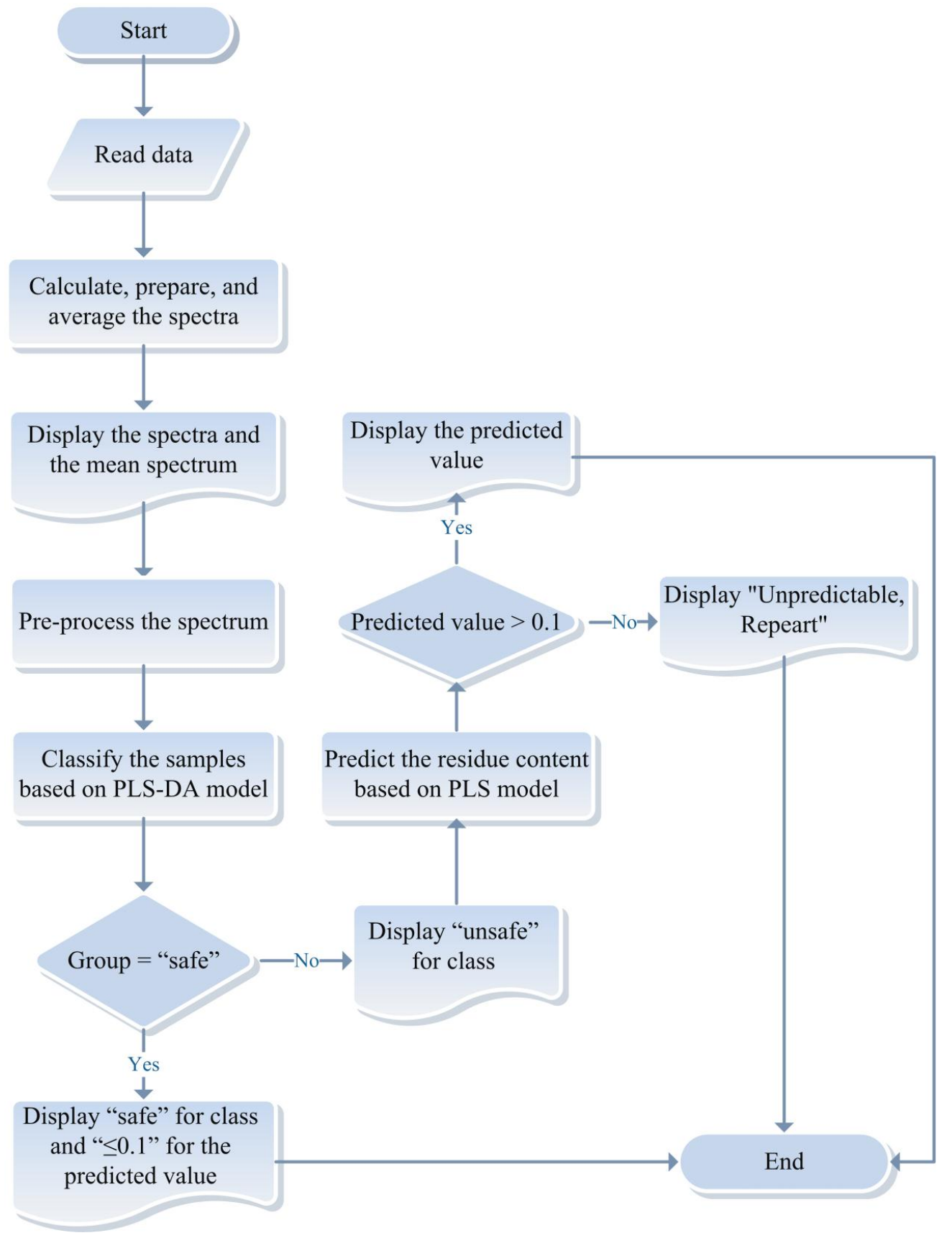

Figure 1. Software flowchart. 


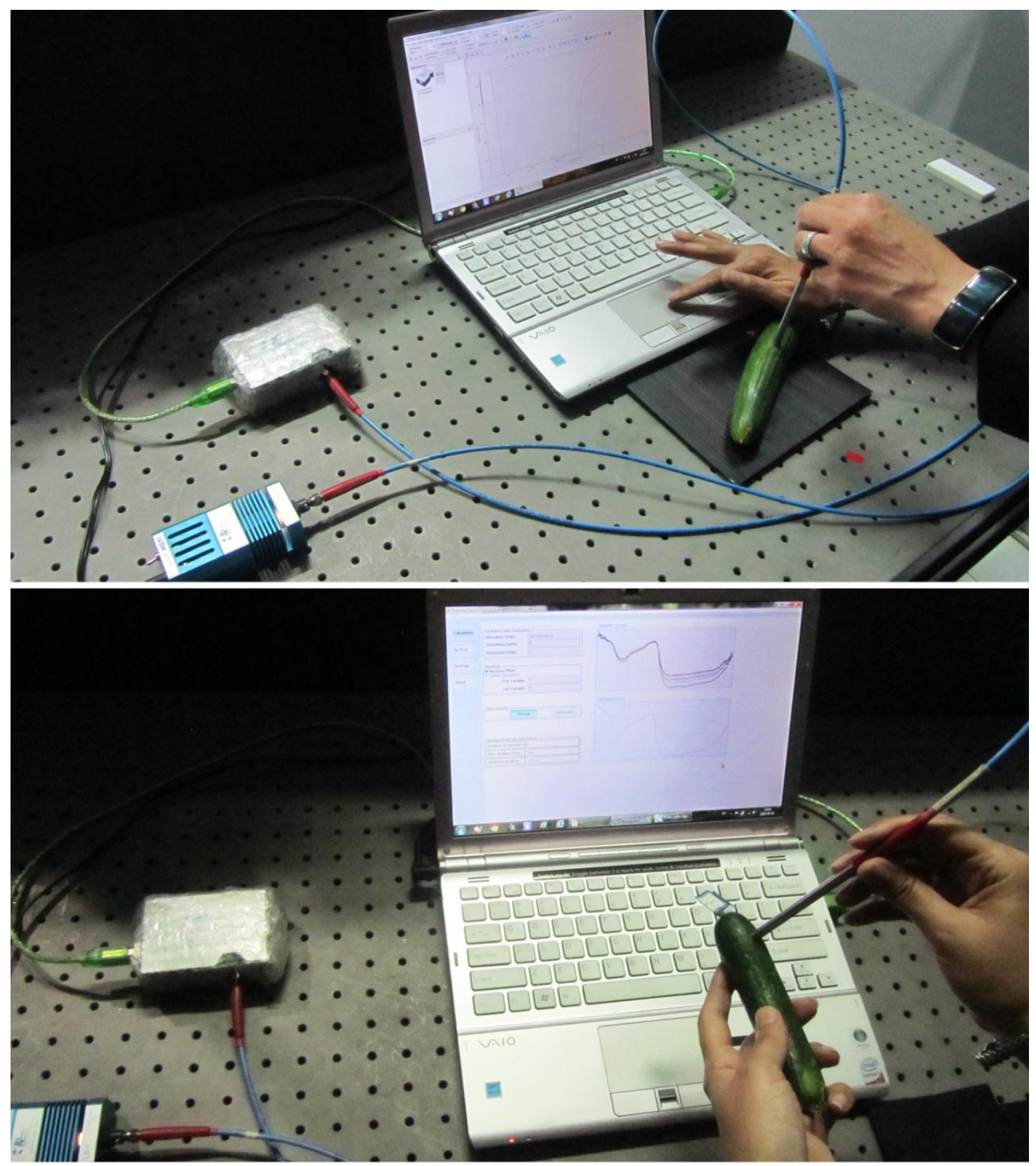

Figure 2. Safety assessment of the samples with the presented Vis/NIR spectroscopic system. 


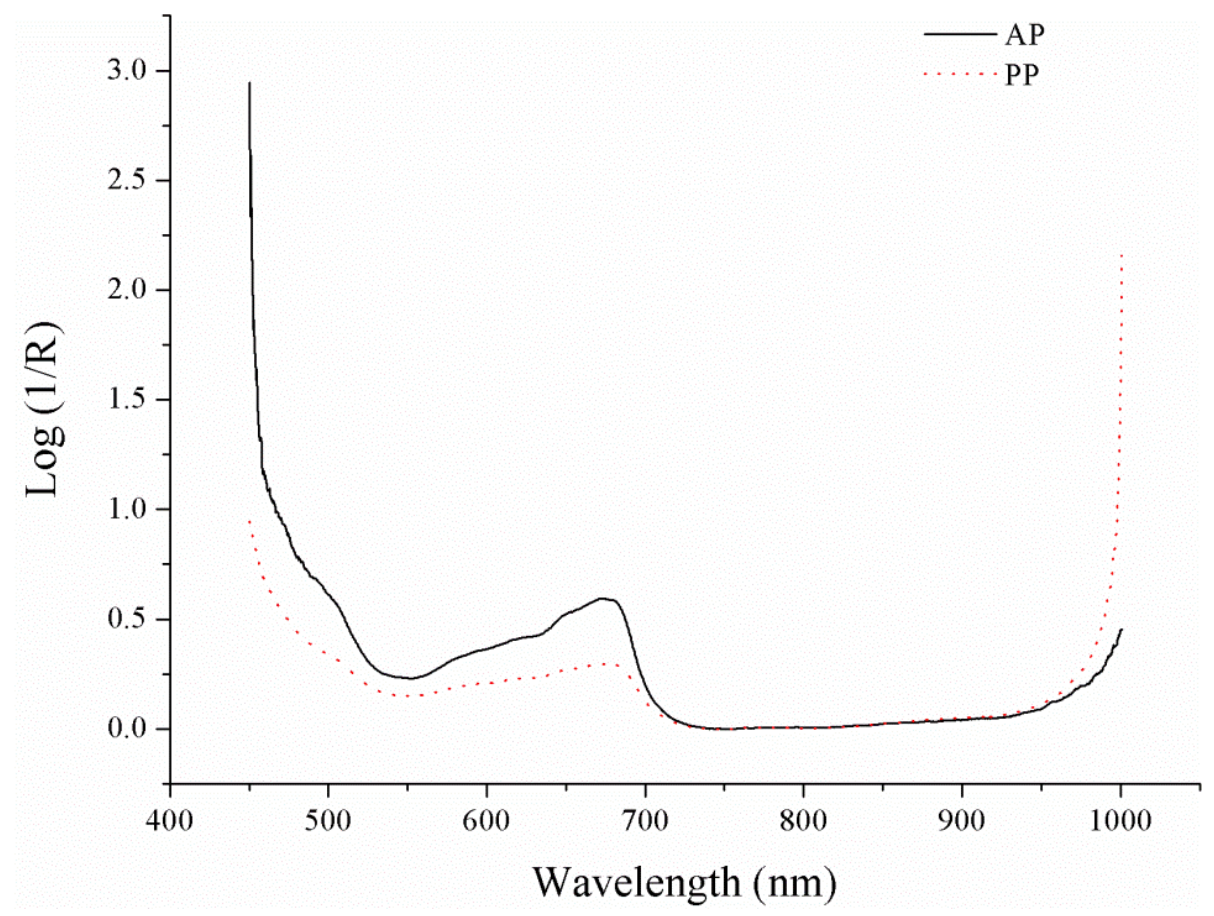

Figure 3. Mean spectra of intact cucumbers with absence of pesticide (AP) and presence of pesticide (PP). 


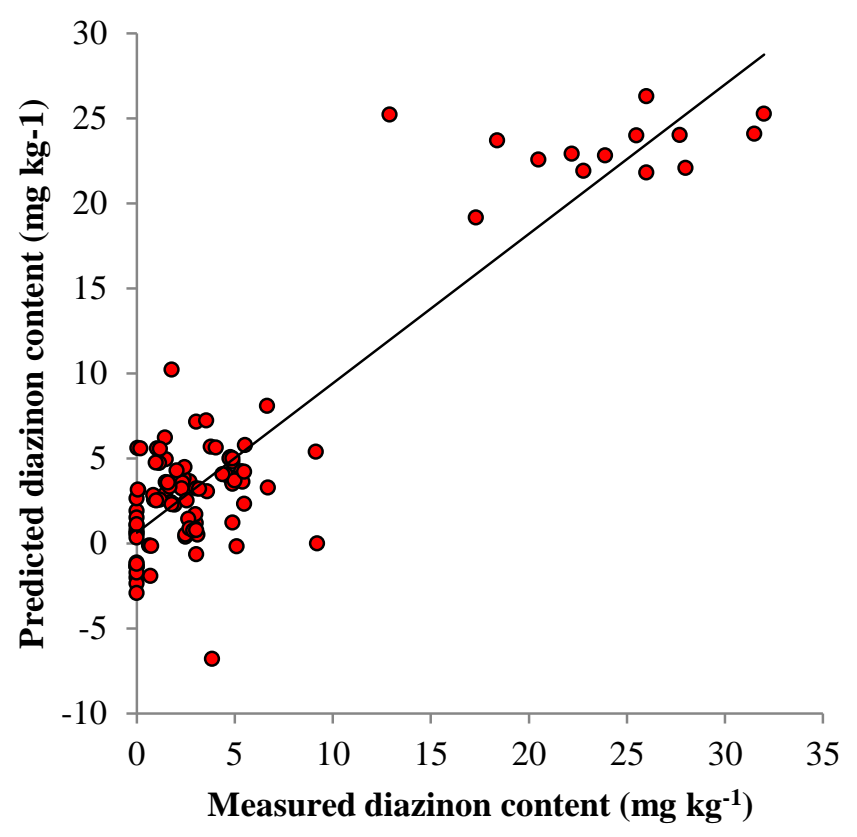

Figure 4. Scatter plot of the correlation between measured and predicted values of diazinon residue in the samples for full cross validation of calibration set. 


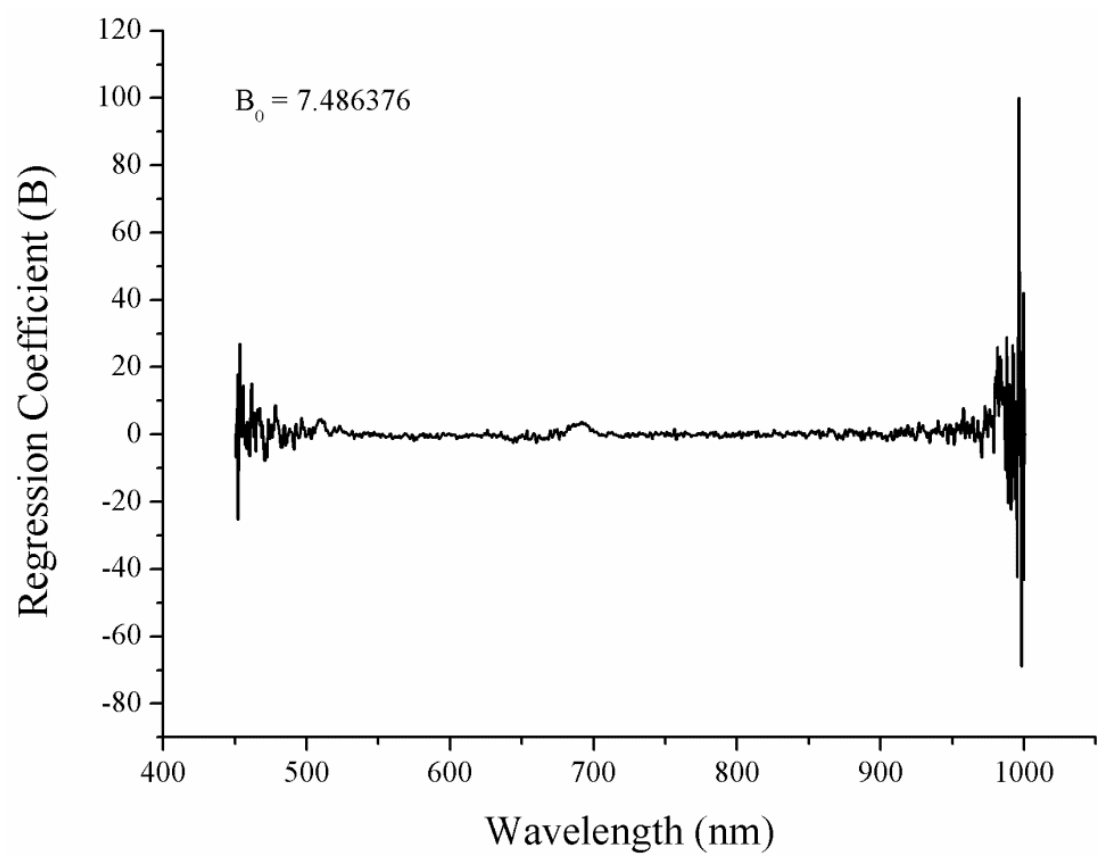

Figure 5. The obtained regression coefficients using the best PLS model developed for diazinon content prediction. 


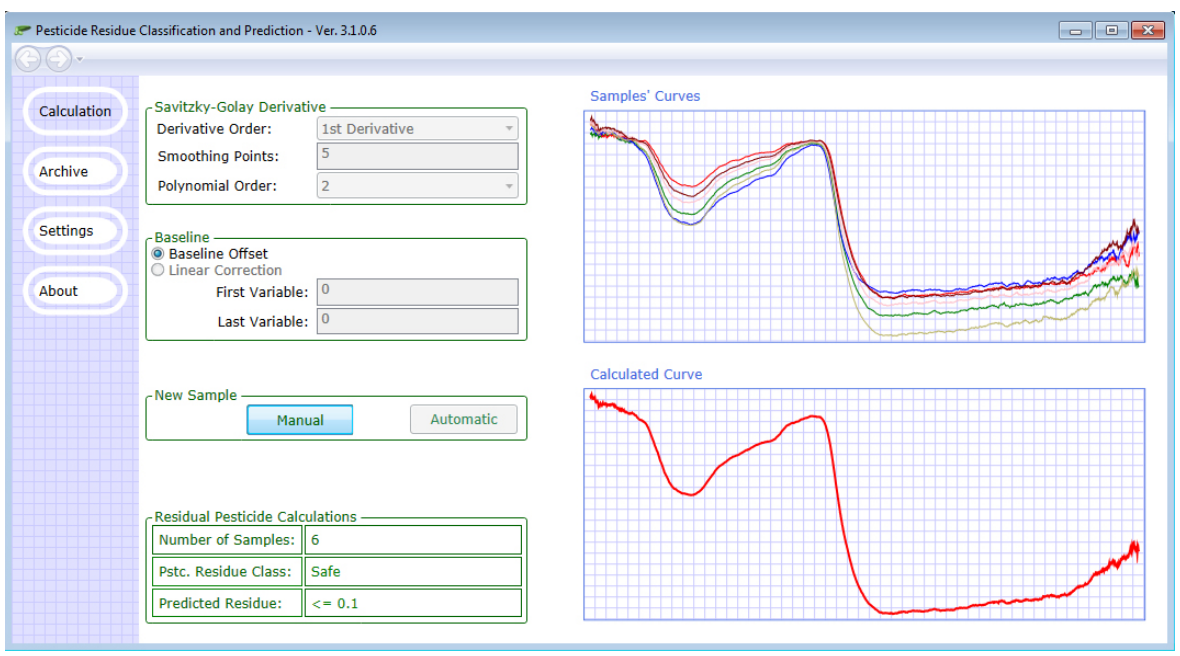

(a)

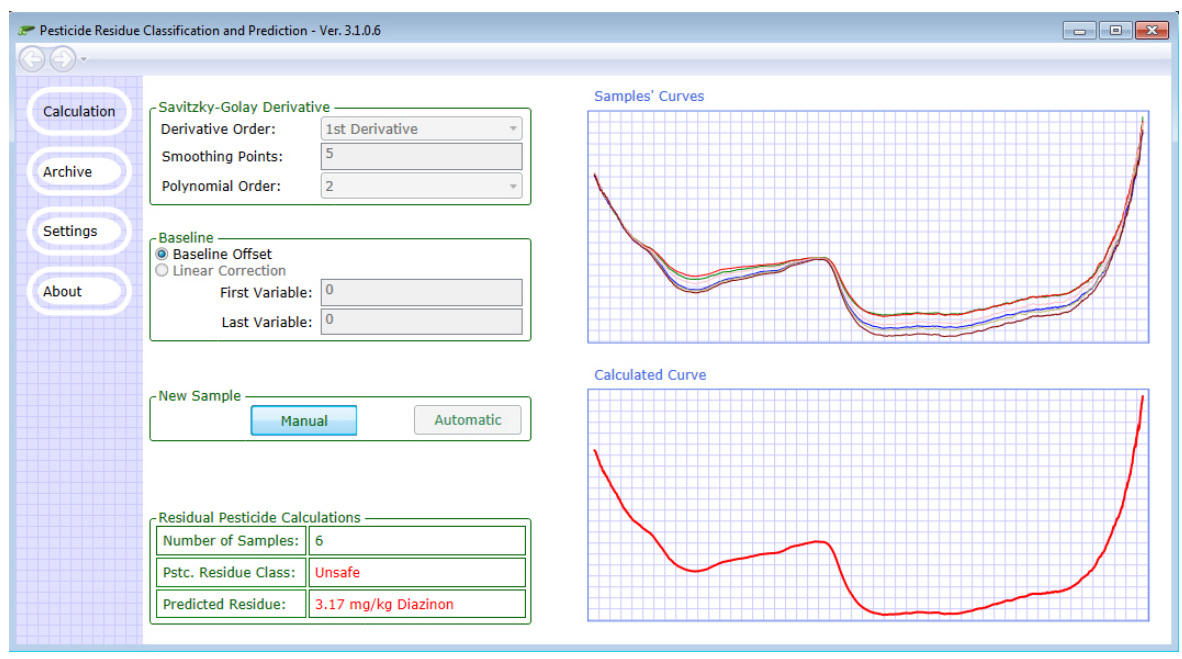

(b)

Figure 6. The performance result of the created GUI for assessment of the typical cucumbers without (a) and with (b) concentrations of diazinon residue. 
Table 1. Statistics of diazinon contents in the cucumbers.

\begin{tabular}{llllllllll}
\hline & & \multicolumn{3}{c}{ Diazinon $\left(\mathrm{mg} \mathrm{kg}^{-1}\right)$} & & & \multicolumn{3}{c}{ Diazinon $\left(\mathrm{mg} \mathrm{kg}^{-1}\right)$} \\
\cline { 3 - 5 } \cline { 7 - 10 } & $\mathrm{N}$ & Range & Mean & SD & Group & N & Range & Mean & SD \\
\hline Total samples & 106 & $0-32$ & 4.85 & 7.44 & Safe & 21 & $0-0.1$ & 0.01 & 0.03 \\
& & & & & Unsafe & 85 & $0.2-32$ & 6.56 & 8.22 \\
\hline
\end{tabular}


Table 2. Results of PLS models for prediction of diazinon residue in the cucumbers.

\begin{tabular}{lccc}
\hline & LV & SECV & $\mathrm{r}_{\mathrm{cv}}$ \\
\hline $\mathrm{MSC}$ & 5 & 3.24 & 0.91 \\
$\mathrm{SNV}$ & 7 & 3.41 & 0.90 \\
$\mathrm{D}_{1}$ & 5 & 4.04 & 0.86 \\
$\mathrm{D}_{2}$ & 6 & 4.69 & 0.81 \\
$\mathrm{MSC}+\mathrm{D}_{1}$ & $\mathbf{3}$ & $\mathbf{3 . 2 2}$ & $\mathbf{0 . 9 1}$ \\
$\mathrm{MSC}+\mathrm{D}_{2}$ & 5 & 3.83 & 0.87 \\
$\mathrm{SNV}+\mathrm{D}_{1}$ & 5 & 3.58 & 0.89 \\
$\mathrm{SNV}+\mathrm{D}_{2}$ & 5 & 4.01 & 0.86 \\
\hline
\end{tabular}

Bold values indicate the best developed model for diazinon prediction in the samples. 
Table 3. Paired t-test results comparing the mean values of diazinon residue predicted using designed system and the values measured by GC analysis.

\begin{tabular}{lccc}
\hline & Mean & SD & SEM \\
\hline Predicted diazinon using designed system $\left(\mathrm{mg} \mathrm{kg}^{-1}\right)$ & 8.39 & 9.32 & 2.26 \\
Measured diazinon using GC analysis $\left(\mathrm{mg} \mathrm{kg}^{-1}\right)$ & 7.36 & 6.09 & 1.48 \\
Difference & 1.03 & 4.67 & 1.13 \\
\hline
\end{tabular}

$$
\mathrm{P}=0.375
$$

$\mathrm{SEM}=$ standard error of mean, $\mathrm{P}=$ probability. 